\title{
Designing iron oxide nanoparticles for image guided thermal medicine applications
}

Hattie L. Ring ${ }^{1,2}$, Sheng Tong ${ }^{3}$, Zhe Gao ${ }^{5}$, Navid Manuchehrabadi ${ }^{5}$, Kaiyi Jiang ${ }^{4}$, Sylvie L. Pailloux $^{1}$, Mark Dresel ${ }^{1}$, Valérie C. Pierre ${ }^{1}$, Christy L. Haynes ${ }^{1}$, Michael Garwood ${ }^{2}$, Gang Bao $^{4}$, John C. Bischof

${ }^{1}$ Department of Chemistry, University of Minnesota, Minneapolis, United States

${ }^{2}$ Center for Magnetic Resonance Research, University of Minnesota, Minneapolis, United States

${ }^{3}$ Department of Biomedical Engineering, University of Kentucky, Lexington, United States

${ }^{4}$ Department of Bioengineering, Rice University, Austin, United States

${ }^{5}$ Department of Mechanical Engineering, University of Minnesota, Minneapolis, United States

E-mail: bischof@umn.edu

\begin{abstract}
The impact of magnetic iron oxide nanoparticle (IONP) core design (size and shape) on magnetic resonance imaging (MRI) and heating characteristics is being actively pursued by many research groups. This work evaluates MRI relaxation and the specific absorption rate $\left(\mathrm{SAR}_{\mathrm{Fe}}\right)$ properties of IONPs as a function of diameter $(6-32 \mathrm{~nm})$. SAR $\mathrm{Fe}$ increased with IONP size up to a maximum of $628 \pm 11 \mathrm{~W} / \mathrm{g}$ Fe for the largest IONP studied $(33 \mathrm{~nm})$. IONPs with a diameter $>10 \mathrm{~nm}$ were demonstrated to have transverse relaxivity $\left(r_{2}\right)$ values suitable for low-concentration IONP imaging applications, such as cell tracking. On the other hand, the optimal IONP diameter for achieving longitudinal relaxivity $\left(r_{1}\right)$ was dependent on magnetic field strength. Measurements of $r_{1}$ values performed at a clinically relevant magnetic field $(1.4 \mathrm{~T})$ indicate all

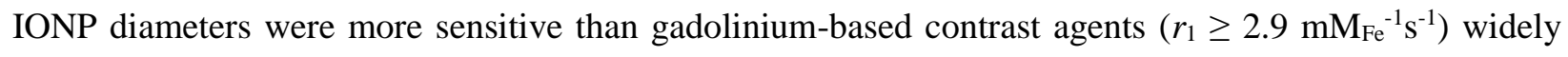
used in clinical imaging. However, such a large $r_{1}$ was not observed when measured at a larger (pre-clinical) magnetic field $(9.4 \mathrm{~T})$. We conclude that the ideal IONP diameter for image guided heating applications is dependent on the magnetic field strength of the MRI for the intended application.
\end{abstract}

\section{Keywords:}

iron oxide nanoparticles, nanoparticle characterization, magnetic relaxation, magnetic fluid hyperthermia, heating, MRI contrast agent 


\section{Introduction}

Magnetic iron-oxide nanoparticles (IONP\$s) have considerable potential as a clinical theranostic platform capable of combining magnetic resonance imaging (MRI) contrast with hyperthermia therapy ${ }^{4-6}$. Clinically, IONPs have been applied for anemia treatment, cancer diagnosis, and are currently in clinical trials for cancer treatment through hyperthermia ${ }^{7}$. Therefore, the impact of IONP design on MRI and heating characteristics continues to be actively explored ${ }^{8-18}$. An important question we consider herein is if a single IONP can be optimized for diagnostic MR imaging and therapeutic heating applications within the same concentration range.

Traditionally, IONP-based MRI contrast agents are used to provide a hypo-intense signal (negative contrast) caused by rapid signal decay from transverse $\left(T_{2}\right)$ relaxation of the water protons ${ }^{19}$. For example, Feridex and Resovist were approved for clinical diagnosis of cancer lesions through negative contrast ${ }^{20,21}$. Similarly, GastroMARK was approved for diagnosis of masses within the gastrointestinal track as an agent that produced negative contrast ${ }^{22}$. Unfortunately, competing imaging methods, gadolinium-based contrast agents (GBCAs) in particular, resulted in the removal of these products from most markets ${ }^{7}$. However, recent safety concerns with GBCAs as contrast agents have resulted in a reemergence of IONP-based contrast agents. Specifically, Feraheme, an anemia treatment, is currently undergoing clinical trials for applications in cancer diagnosis (pancreatic, bone, and metastatic), central nervous system disorders, and angiography ${ }^{7}$. All of these applications focus on the use of echo-based pulse sequences, such as gradient echo and spin echo sequences. The negative contrast produced with echo-based MR imaging methods is capable of localizing IONPs and can quantify IONPs existing in low concentrations $\left(\leq 0.018 \mathrm{mM}_{\mathrm{Fe}}\right)$ through the measurement of the transverse relaxation rate constant, $R_{2}=1 / T_{2}{ }^{23}$. Recent developments of very small IONPs and echoless pulse sequences have produced positive contrast with longitudinal $\left(T_{1}\right)$ relaxation ${ }^{19,24}$. Positive contrast can provide IONP concentration at higher concentrations $\left(<60.7 \mathrm{mM}_{\mathrm{Fe}}\right)$ through the measurement of the longitudinal relaxation rate constant, $R_{1}=1 / T_{1}{ }^{4,19,25,26}$.

Relaxation rates as a function of IONP concentration are known as relaxivities $\left(r_{1}\right.$ or $\left.r_{2}\right)$. Relaxivities are defined as the slope of the linear regression of the relaxation rate constant $\left(R_{\mathrm{x}}\right.$, where $\left.\mathrm{x}=1,2\right)$ and the concentration of the contrast agent ([IONP]):

$$
R_{x}=R_{x 0}+r_{x} \cdot[I O N P]
$$

where $R_{\mathrm{x} 0}$ is the relaxation rate constant of the media without the IONP and $r_{\mathrm{x}}$ is the relaxivity. Positive $\left(T_{1^{-}}\right.$ weighted) contrast is obtainable with echo-based MRI sequences when the contrast agent has a low $r_{2} / r_{1}$ ratio (e.g., $\left.r_{2} / r_{1}=2\right)^{24}$. However, the ability to produce positive contrast with echo-based MRI sequences is limited for commercially available IONPs with typical ratios $r_{2} / r_{1}=59-1429{ }^{26}$. Recent work has demonstrated an ability to perform positive contrast IONP quantification, even at high $r_{2} / r_{1}$ ratio and high IONP concentration (as high as $60.7 \mathrm{mM}_{\mathrm{Fe}}=3.4 \mathrm{mg} \mathrm{Fe} / \mathrm{mL}$ ), through the use of ultra-short echo or echoless pulse sequences, such as sweep-imaging with Fourier transform (SWIFT), which measure $R_{1}{ }^{19,27-30}$.

Magnetic fluid heating (MFH) is a technique where heat, measured as the specific absorption rate $\left(\mathrm{SAR}_{\mathrm{Fe}}\right)$, is produced by the interaction between an alternating magnetic field and IONPs ${ }^{31,32}$. MFH has been applied to both hyperthermia cancer therapy and nanowarming of cryopreserved tissues $5,12,33,34$. Both applications benefit from a good estimate of thermal dose to avoid unintentional injury to surrounding tissues. Magnetic particle imaging (MPI) shows great promise as a means to image IONP localization and control heating, 
but it is still under development for use in the clinic ${ }^{35,36}$. Computed tomography (CT) is capable of imaging high IONP concentrations (> $18 \mathrm{mM}_{\mathrm{Fe}}, 1 \mathrm{mg} \mathrm{Fe} / \mathrm{mL}$ ); however, as is the case with the high heating IONPs described herein, concentrations below $1 \mathrm{mg} \mathrm{Fe} / \mathrm{mL}$ are capable of causing unintended tissue damage through heating ${ }^{37}$. Measurement of $R_{1}$ through echoless pulse sequences have been shown to correlate linearly with thermal response in agarose samples and tissues ${ }^{4,25,38}$.

The mechanisms by which IONPs produce heat via MFH or cause MR relaxation are different, although both relate to the fundamental magnetic properties of the IONP. MFH produces heat by exposing IONPs to a $\mathrm{kHz}$ magnetic field in the absence of a background magnetic field. The applied alternating magnetic field causes the magnetization of the IONP to change orientation, whereas the amount of heat produced is dependent on the magnetic hysteresis of the material at the applied AC magnetic field strength and frequency ${ }^{39,40}$. From a simplified view, MRI is a spectroscopic technique exploiting the Zeeman energy splitting of protons created with a static magnetic field, typically $\geq 1 \mathrm{~T}$. MRI signal is generated by applying a radio frequency pulse in the $\mathrm{MHz}$ range. In the static magnetic field used in MRI, the IONP is magnetically saturated and therefore will not respond to the $\mathrm{kHz}$ magnetic fields typically applied during MFH. On the other hand, the presence of the IONP accelerates the MRI relaxation processes. Certain physical properties, like magnetic susceptibility, affect both MRI relaxation and heating; therefore, a single particle might be designed to perform well for both of these applications.

Many IONPs have been developed and assessed for both MRI contrast and MFH ${ }^{4,9,19,25,38,41-43}$; however, these applications are typically considered separately and not within the same concentration range. Additionally, IONP size is known to have an impact on magnetic properties impacting both MRI and MFH 9,18 . Herein, we take a comparative look at the heating and imaging properties of IONPs as a function of IONP core size while maintaining a faceted structure. There results herein enable us to optimize IONP size for the application of magnetic resonance image guided magnetic fluid hyperthermia.

\section{Results \& Discussion}

\section{Heating}

Previous work demonstrated that larger diameter IONPs $(33 \mathrm{~nm})$ produced $\mathrm{SAR}_{\mathrm{Fe}}$ that is much higher than that of other published IONPs $2,9,14,41$. This observed high heating is attributed to the stability from the DSPE-PEG2000 coating and the ferromagnetic nature of these IONPs ${ }^{9}$. Analysis of all IONP diameters was performed at the University Minnesota with a lower frequency setting (20 kA/m and $190 \mathrm{kHz})$ to allow a direct comparison with heating measurements performed with commercially available Ferrotec EMG-308 ${ }^{25}$. The $\mathrm{SAR}_{\mathrm{Fe}}$ was observed to increase dramatically between $11-30 \mathrm{~nm}$, similar to the trend described by Tong et. al. (Figure 1) ${ }^{9}$. The change in experimental parameters, such as background matrix, magnetic field strength, and frequency, explain the observed lowered $\mathrm{SAR}_{\mathrm{Fe}}{ }^{2,39}$. Furthermore, the $\mathrm{SAR}_{\mathrm{Fe}}$ observed for the $32 \mathrm{~nm}$ diameter IONPs is three times higher than commercially available IONPs, such as EMG-308 (Ferrotec), BNF-Starch (Micromod), nanomag ${ }^{\circledR}$-D-spio (Micromod), and Feridex (Advanced Magnetics) ${ }^{14,25}(\mathrm{p}<0.001)$. The increased $\mathrm{SAR}_{\mathrm{Fe}}$ allows for a reduction in necessary IONP concentration for magnetic fluid hyperthermia and nanowarming applications.

\section{Transverse Relaxivity $\left(r_{2}\right)$}

At the preclinical $(9.4 \mathrm{~T})$ field strength, the $r_{2}$ is observed to increase with IONP diameter. A similar trend was observed at the clinical $(1.4 \mathrm{~T})$ field strength; however, a plateau was measured for IONP diameters 
above $10 \mathrm{~nm}$ (Figure 2). Furthermore, $r_{2}$ is anticipated to be constant with magnetic field strength because the saturation magnetization for each IONP is far below $(<5 \mathrm{mT}) 1.4 \mathrm{~T}^{18}$. The differences observed between 1.4 and $9.4 \mathrm{~T}$ can be attributed to the difference in temperature for the experimental setup (37 and $22{ }^{\circ} \mathrm{C}$, respectively). The temperature dependence of $r_{2}$ has been demonstrated to be stronger for larger IONP diameters ${ }^{3,18}$.

The $r_{2}$ values shown at $1.4 \mathrm{~T}$ for diameter $>6 \mathrm{~nm}$ demonstrate an $r_{2}$ which is larger and therefore has an improved sensitivity when compared with EU-approved Resovist $\left(61 \mathrm{mM}_{\mathrm{Fe}^{-1}} \mathrm{~s}^{-1}\right)$ and FDA-approved Feridex $\left(41 \mathrm{mM}_{\mathrm{Fe}^{-1}} \mathrm{~s}^{-1}\right)^{3}$. In addition to applications as an MRI contrast agent, IONPs with a high $r_{2}$ are of particular interest at low concentrations for labeling molecular markers for disease diagnostic MRI. Molecular marker applications measure the migration of cells over time by loading the cells with IONPs before injection ${ }^{44}$. In general, $r_{2} \geq 324 \mathrm{mM}_{\mathrm{Fe}}{ }^{-1} \mathrm{~s}^{-1}$ is considered a reasonable relaxivity to facilitate the measurement of single cells ${ }^{45}$. However, higher $r_{2}$ is better for molecular marker applications because the necessary IONP uptake to allow cell tracking is reduced ${ }^{41}$. Therefore, IONPs $>6 \mathrm{~nm}$ would be feasible for cell tracking at $1.4 \mathrm{~T}$ and highly applicable at preclinical field strengths $(9.4 \mathrm{~T})$.

\section{Longitudinal Relaxivity $\left(r_{1}\right)$}

Gadolinium-based contrast agents are used clinically to provide positive ( $T_{1}$-based) contrast with typical $r_{1}$ values ranging from $2.9-4.7 \mathrm{mM}^{3}$. In general, IONPs often are capable of providing more sensitivity with a higher $r_{1}$. However, their clinical use is limited to $r_{2}$ due to their higher $r_{2} / r_{1}$ ratio (see Table S1). Some IONPs have been specifically designed to mimic the $r_{2} / r_{1}$ ratio $(\sim 1)$ of GBCAs to produce positive contrast 3. Similar $r_{2} / r_{1}$ ratios (2) have only been demonstrated for IONPs with very small diameters $(3 \mathrm{~nm})$. These IONPs have low $r_{1}\left(5.2 \mathrm{mM}_{\mathrm{Fe}}{ }^{-1} \mathrm{~s}^{-1}\right)$ and $r_{2}\left(10.5 \mathrm{mM}_{\mathrm{Fe}^{-1}} \mathrm{~s}^{-1}\right)$, impacting their sensitivity ${ }^{24}$, although they remain comparable to clinical GBCAs.

Ultrashort-echo and echoless pulse sequences have been developed with zero or negligible acquisition delay, allowing for the detection of signals with rapid $T_{2}$ relaxation to obtain $T_{1}$-weighted images 4,18,19,29,30,46-48. Acquisition of $T_{1}$-weighted images allows for IONP design which can focus on optimization of $r_{1}$. At $9.4 \mathrm{~T}$, the echoless sequence SWIFT has demonstrated a quantitative range from 0.1 to $35.7 \mathrm{mM}_{\mathrm{Fe}}$ (0.05 to $3.0 \mathrm{mg}_{\mathrm{Fe}} / \mathrm{mL}$ ) using $R_{1}\left(=1 / T_{1}\right)$ mapping ${ }^{26}$.

The comparison between $r_{1}$ acquired at 1.4 and 9.4 T demonstrates a much lower $r_{1}$ at the higher magnetic field (Figure 3). Of note, at $1.4 \mathrm{~T}$, IONP core sizes $>10 \mathrm{~nm}$ maintain an $r_{1}$ above values typically observed for clinical GBCAs at $1.5 \mathrm{~T}^{3}$. The observed change in $r_{1}$ with magnetic field strength is consistent with theory described by Gillis et. al. and observations by Smolensky et. al. ${ }^{18,49}$. As higher magnetic fields are applied, one of the mechanisms of relaxation, Néelian relaxation, is inhibited because the magnetic moment of the IONP is locked into the same orientation as the main magnetic field. Therefore, the relaxation rate constant and relaxivity decreases with field strength ${ }^{50}$.

At both field strengths, we observe a local maximum and then a decrease in $r_{1}$ with IONP diameter (Figure 3). The change between the values at high field strength is small enough $\left(0.2-0.8 \mathrm{mM}_{\mathrm{Fe}^{-1}} \mathrm{~s}^{-1}\right)$ to be considered invariant. Smolensky et. al. compared faceted IONPs with spherical IONPs demonstrating different trends ${ }^{18}$. Our IONPs have a different surface coating for stabilization, but were observed to be faceted (see Figure S1). Our IONPs follow a trend similar to the faceted IONPs synthesized by Smolensky et. al. within IONP diameters of $4-18 \mathrm{~nm}$. However, the increase in $r_{1}$ with IONP diameter is no longer

maintained once ferromagnetic character is exhibited. The ferromagnetic character for the IONP 
synthesized has been demonstrated previously for diameters larger than $18 \mathrm{~nm}$ based on magnetic hysteresis characterization ${ }^{9}$. Therefore, the decreasing trend observed at $9.4 \mathrm{~T}$ for IONPs $>18 \mathrm{~nm}$ matches the expectations for an IONP with a high anisotropy as is observed with ferromagnetic particles ${ }^{18,51}$.

\section{Heating and Relaxivity Characteristic Relationship}

Our results provide the capability to evaluate the correlation between $\mathrm{SAR}_{\mathrm{Fe}}$ and $r_{1}$ or $r_{2}$. The SAR $\mathrm{Fe}_{\mathrm{Fe}}$ has been demonstrated to have a direct correlation with IONP diameter, independent of the change in field strengths used by Tong et. al. and within this manuscript ${ }^{9}$. Similarly, $r_{2}$ demonstrates an increasing trend with IONP diameter despite the background magnetic field. In contrast, the IONP diameter which corresponds to the maximum $r_{1}$ shifts with magnetic field strength. Therefore, a correlation between $\mathrm{SAR}_{\mathrm{Fe}}$ and $r_{2}$ is conceivable and worth further consideration.

The dependence on $\mathrm{SAR}_{\mathrm{Fe}}$ and $r_{2}$ on experimental parameters, such as magnetic field strength and frequency, create challenges for directly comparing results with other research groups. Our dataset demonstrates the challenge regarding the changes in $r_{2}$ with field strength and differences in $\mathrm{SAR}_{\mathrm{Fe}}$ of the same IONP measured at both the University of Minnesota (Figure 1) and Rice University ${ }^{9}$. Bonvin et. al. and Mohapatra et. al. focused on changes in IONP crystalline and shape properties, respectively, and indicate a potential direct correlation between $\mathrm{SAR}_{\mathrm{Fe}}$ and $r_{2}{ }^{41,42}$. However, the direct numerical comparison is complicated by changes in the experimental setup and IONP synthesis and, therefore, magnetic characteristics. Changes in IONP crystal structure cannot be observed through SQUID, Mössbauer spectroscopy, TEM, or x-ray diffraction analysis, but can be measured with small angle neutron scattering have been demonstrated to still have an impact on $\mathrm{SAR}_{\mathrm{Fe}}{ }^{52}$. Furthermore, changes in surface anisotropy caused by the attachment of a surface coating are known to impact both $\mathrm{SAR} F$ and $r_{2}{ }^{8,38,53}$. Therefore, a robust demonstration of the correlation between $\mathrm{SAR}_{\mathrm{Fe}}$ and $r_{2}$ requires a comprehensive study which must incorporate small angle neutron scattering to fully characterize the shape and crystalline structure of the IONP; that study is beyond the scope of work presented here and will be the subject of future research.

\section{$\mathrm{R}_{1}$ Image Guided Heating}

Several groups have demonstrated multifunctional IONPs capable of both heating and imaging; however, the imaging capability focused on negative $T_{2}$-weighted detection ${ }^{41,42}$. Additionally, others have reported $r_{1}$ properties; however, they do not endeavor to use both heating and imaging within the same concentration range ${ }^{43}$. Herein, our goal is to analyze IONPs using $R_{1}$ mapping to provide quantitative information within a reasonable IONP concentration for magnetic fluid hyperthermia ${ }^{4}$. The heightened heating produced by these IONPs provide an improved overlap in IONP concentration range of $R_{1}$ mapping and measurable heating.

The feasibility of using an $R_{1}$ map to predict the expected temperature change after 3 minutes of heating is shown in Figure 4. The change in temperature observed for samples at concentrations above $16 \mathrm{mM}_{\mathrm{Fe}}(0.896$ $\mathrm{mg} \mathrm{Fe} / \mathrm{mL}$ ) are high enough to damage tissues ${ }^{1,2}$. Agarose samples are a simplification compared to the complex milieu of the intended biological systems for magnetic fluid hyperthermia. However, previous work has demonstrated the ability to work within an in vivo system to create a calibration between an $R_{1}$ map and heating capabilities ${ }^{4}$. Therefore, this simplified system informs the size selection of this IONP before moving forward to pre-clinical studies connecting imaging and heating parameters. 
Due to the $r_{1}$ field dependence, the ideal IONP diameter is dependent on the applied field strength. At the preclinical field strength $(9.4 \mathrm{~T})$, IONP diameters of 19 and $33 \mathrm{~nm}$ were observed to have such a low $r_{1}$ that the change in $R_{1}$ based on IONP concentration is negligible. Therefore, a heating prediction cannot be ascertained, and a trade-off is necessary between selecting the highest heating IONP (32 nm, which has a very low $\left.r_{1}\right)$ and a low-heating IONP $\left(6 \mathrm{~nm}\right.$, which has an acceptable $\left.r_{1}\right)$. At the clinically relevant field strength $(1.4 \mathrm{~T})$, the heating does not need to be sacrificed for better relaxation sensitivity because all of the IONP diameters measured had a sufficiently high $r_{1}$ to obtain adequate sensitivity for imaging. Thus, for MRI applications at $1.4 \mathrm{~T}$, IONP diameter could be optimized based on heating properties.

\section{Conclusions}

This work represents the characterization of IONPs toward an optimization for $T_{1}$-weighted MR imaging and MFH. IONPs were observed to provide increased heating as the diameter increased, with IONPs with a $33 \mathrm{~nm}$ diameter demonstrating three times more heat than commercially available IONPs. In contrast, IONP cores $>10 \mathrm{~nm}$ had sufficiently high $r_{2}$ to provide utility for MRI detection in low concentration, as needed in cell tracking. Further, the $r_{1}$ values measured at the clinically relevant field $(1.4 \mathrm{~T})$ were larger than the $r_{1}$ values of GBCAs widely used in clinical imaging. However, such a large $r_{1}$ value was not observed at the pre-clinical field (9.4 T). In summary, IONPs with satisfactory heating and MR imaging performance have been demonstrated herein, however; there remains future opportunities to develop IONPs with optimized performance for both types of applications.

\section{Methods}

\section{Materials}

Iron acetylacetonate $\left(\mathrm{Fe}(\mathrm{acac})_{3}, 99 \%\right)$, 1,2-tetradecandiol (technical grade, 90\%), oleic acid (technical grade, 90\%), oleylamine (technical grade, 70\%), benzyl ether (98\%), hydrochloric acid (> 37\%), hydroxylamine $\mathrm{HCl}$, sodium hydroxide, ammonium acetate, and agarose (CAS:9012-36-6) were purchased from Sigma-Aldrich (St. Louis MO) and used as received. All water used was MilliQ filtered water.

\section{IONP Synthesis and Characterization}

Magnetite nanocrystals were synthesized and characterized as previously described ${ }^{9}$. XRD and heating characterization were not anticipated to change significantly between batches. Water-dispersible IONPs were generated by coating the magnetite nanocrystals with DSPE-PEG 2000 using a dual solvent exchange method. Table 1 lists the specific IONP sizes investigated and the corresponding size and polydispersity. Size characterization was performed on each batch of IONPs using transmission electron microscopy (TEM, Figure S1). TEM images were acquired using a transmission electron microscope (Hitachi H-7500) at the Rober P. Apkarian Integrated Electron Microscopy Core at Emory University and a transmission electron microscope (JEOL 1230) at Rice University. The average diameter of the nanocrystals was calculated based on the measurements of at least 300 nanocrystals using image processing software (ImagePro Plus). The iron concentration of the IONPs was determined based on the Ferrozine assay with validation using ICP-OES ${ }^{54}$. Before iron concentration determination, the IONPs were digested in $0.3 \mathrm{M}$ hydrochloric and ascorbic acid for two hours at $60{ }^{\circ} \mathrm{C}$. ICP-OES of the iron concentration was performed with a Thermo Scientific iCAP 6500 dual-view ICP-OES (West Palm Beach, FL, USA) with 1150 W power. 


\section{Sample Preparation}

Heating and 9.4 $\mathrm{T}$ relaxivity measurements were performed with IONPs suspended in $1 \%$ agarose at concentrations ranging from 0.018 to $89.3 \mathrm{mM}_{\mathrm{Fe}}(0.001$ to $5 \mathrm{mg} \mathrm{Fe} / \mathrm{mL})$. $1.4 \mathrm{~T}$ relaxivity measurements were performed with IONPs suspended in water at concentrations ranging from 0.071 to $8.9 \mathrm{mM}_{\mathrm{Fe}}(0.004$ to $0.500 \mathrm{mg} \mathrm{Fe} / \mathrm{mL}$ ). The lower concentrations of IONP were necessary at $1.4 \mathrm{~T}$ due to hardware limitations related to the measurement of rapidly decaying spins. Heating measurements were performed on all but the smallest IONPs because those below $10 \mathrm{~nm}$ produce negligible heat ${ }^{9}$.

\section{Heating Characterization}

A $1 \mathrm{~kW}$ Hotshot inductive heating system with a 2.75-turn, water-cooled copper coil (Ameritherm Inc., Scottsville, NY) was used to generate the alternating magnetic field. Reported $\mathrm{SAR}_{\mathrm{Fe}}$ data were acquired at $20 \mathrm{kA} / \mathrm{m}$ field strength (volume-averaged peak-amplitude across the sample) with an applied frequency fixed at $190 \pm 10 \% \mathrm{kHz}{ }^{25,38}$. $\mathrm{SAR}_{\mathrm{Fe}}$ was measured for IONP concentrations ranging from $1.8-89.3 \mathrm{mM}_{\mathrm{Fe}}$ $(0.1-5 \mathrm{mg} \mathrm{Fe} / \mathrm{mL})$. Each $1 \mathrm{~mL}$ sample in a plastic microcentrifuge tube was insulated and centered in the inductive coil and heated for 3 minutes with the temperature recorded every second with a T/Guard 405 Fiber Optic Monitoring System (Qualitron, Quebec, Canada). The SAR was calculated using Box-Lucas fitting for $55 \mathrm{~s}$ after heating began.

Validation of the high heating properties of the $40 \mathrm{~nm}$ IONPs was conducted at the University of Minnesota using settings and analysis consistent with practices at Rice University. The $\mathrm{SAR}_{\mathrm{Fe}}$ was acquired in water at $20 \mathrm{kA} / \mathrm{m}$ and $360 \mathrm{kHz}$ by calculating with the Time-rise fitting for $20 \mathrm{~s}$ after heating began. At the University of Minnesota, the $\mathrm{SAR}_{\mathrm{Fe}}$ was measured to be $2125 \pm 73 \mathrm{~W} / \mathrm{g} \mathrm{Fe}$, slightly lower than the 2560 $\mathrm{W} / \mathrm{g}$ Fe reported in Tong et. al. ${ }^{9}$. The slight difference in heating characterization is likely attributed to small differences in laboratory protocol and experimental setup (i.e. the uniformity of the field, higher harmonics in AC field, or heat losses within the system) between the labs at Rice University and University of Minnesota ${ }^{55}$, but the measured $\mathrm{SAR}_{\mathrm{Fe}}$ is extraordinary compared to previous work with other IONPs. Unfortunately, the $40 \mathrm{~nm}$ IONP was unstable when moved into the $1 \%$ agarose medium and could not be compared directly with the data demonstrated herein.

\section{Relaxivity Characterization at $9.4 T$}

MRI, including $R_{1}$ and $R_{2}$ measurement, was performed with a $9.4 \mathrm{~T}-31 \mathrm{~cm}$ bore MRI scanner (Agilent Technologies, Santa Clara, CA). All images were acquired with a volume transmit/receive coil having an inner diameter of $3 \mathrm{~cm}$ (Agilent Technologies, Santa Clara, CA).

A multi-slice $T_{2}$-weighted spin echo (SE) sequence was used to measure $R_{2}$ maps. Each 2D image was acquired with a repetition time (TR) of $2.4 \mathrm{~s}$, acquisition bandwidth of $50 \mathrm{kHz}$, an acquisition time of 2.56 $\mathrm{ms}$, a slice thickness of $5 \mathrm{~mm}$, and a resolution of 417 x $417 \mu \mathrm{m}$. For $R_{2}$ mapping, the echo time (TE) was arrayed across 16 points spaced logarithmically between 12 and $800 \mathrm{~ms}$. All 2D images were reconstructed using VnmrJ versions 3.2.

3D $T_{1}$-weighted images and $R_{1}$ maps were acquired using a Look-Locker method together with a MultiBand (MB)- sweep imaging Fourier transform (SWIFT) sequence for readout. The MB-SWIFT flip angle was $1^{\circ}$, acquisition delay $\approx 2 \mu \mathrm{s}$, acquisition bandwidth $=384 \mathrm{kHz}, \mathrm{TR}=1.2 \mathrm{~ms}$, gaps $=2$, voxel resolution $=$ $194 \times 194 \times 1172 \mu \mathrm{m}$, and total acquisition time $\sim 7 \mathrm{~min}^{19,48}$. The field-of view was $50 \times 50 \times 150 \mathrm{~mm}^{3}$ with image matrix size $=128 \times 128 \times 128 \times 64(\mathrm{x}, \mathrm{y}, \mathrm{z}, \mathrm{t})$. Two different MB-SWIFT settings, which impact the 
time points acquired, were also adjusted $\left(\mathrm{N}_{\text {spiral }}=128\right.$ and 32 and $\mathrm{N}_{\mathrm{v}}=1024$ and 4096, respectively). The $\mathrm{N}_{\mathrm{v}}=1024$ setting had 64 time points spaced linearly from 12.7 to $1152 \mathrm{~ms}$. The $\mathrm{N}_{\mathrm{v}}=4096$ setting had 64 time points spaced linearly from 39.8 to $4596 \mathrm{~ms}$. MB-SWIFT images were reconstructed using an in-house program written in MATLAB $(2012 b)^{48}$.

For each voxel, the time points were fit to the exponential relaxation curve using a three-variable fit ${ }^{19}$. The relaxation rate constants $\left(R_{1}\right.$ or $\left.R_{2}\right)$ were determined with least-squares fitting. The region of interest (ROI) assessed for each tube was approximated as a cuboid with dimensions 2.05 x 2.05 x $5 \mathrm{~mm}$. For SE, a 6 x 6 voxel square ROI was selected in the center of each tube for a single slice. For MB-SWIFT, the $12 \times 12$ voxel square ROI was selected in the center of each tube across 4 slices. The relaxivity $\left(r_{1}\right.$ or $\left.r_{2}\right)$ was determined by performing a linear least-squares fitting of the relaxation rates as a function of iron concentration.

\section{Relaxivity Characterization at $1.4 T$}

Benchtop relaxometry measurements were performed with a Bruker Minispec mq60 NMR Analyzer (Billerica, MA) at $60 \mathrm{MHz}$. The temperature was controlled at $37{ }^{\circ} \mathrm{C}$ using a Julabo F25 circulating water bath. Both relaxations were acquired using scan $=2, T R=4 \mathrm{~s}$; and acquisition bandwidth $=20 \mathrm{kHz}$. Relaxation rate constants were determined with monoexponential curve fitting and relaxivity analyses were performed in triplicate and averaged. $R_{1}$ values were obtained via an inversion recovery sequence with 20 acquisition points. $R_{2}$ values were obtained with a Carr-Purcell-Meiboom-Gill sequence with 20,000 acquisition points.

\section{Abbreviations}

IONP: iron-oxide nanoparticle; $\mathrm{SAR}_{\mathrm{Fe}}$ : specific absorption rate; MRI: magnetic resonance imaging; $\mathrm{MFH}$ : magnetic fluid hyperthermia; $\mathrm{HCl}$ : hydrochloric acid; TEM: transmission electron microscopy; ICP-OES: inductively coupled plasma optical emission spectroscopy; SE: spin echo; TR: repetition time; TE: echo time; MB-SWIFT: multi-band sweep imaging with Fourier transform; ROI: region of interest; GBCA: gadolinium-based contrast agents.

\section{Acknowledgements}

This work was supported in part by the Cancer Prevention and Research Institute of Texas (RR140081 to G.B.) and by NIH R01 HL135046, NIH R01 DK117425, and NIH P41 EB015894.

\section{Contributions}

Hattie L. Ring wrote the manuscript, coordinated the study, acquired and analyzed the $9.4 \mathrm{~T}$ relaxation data, analyzed the $1.4 \mathrm{~T}$ relaxivities, and analyzed heating data. Sheng Tong developed, synthesized, and characterized the IONPs. Zhe Gao coordinated transportation of samples and acquired and analyzed heating data. Navid Manuchehrabadi acquired heating data. Kaiyi Jiang synthesized IONPs. Sylvie L. Pailloux and Mark Dresel measured and analyzed 1.4 T relaxation rates. Valérie C. Pierre provided use of the Bruker mq60 relaxometer and expertise in contrast agents. Christy L. Haynes provided chemistry wetlab space and her insight on nanoparticle characterization. Michael Garwood provided knowledge regarding magnetic resonance imaging, help with study design, access to the $9.4 \mathrm{~T}$ for relaxivity measurements, and funding to cover the imaging cost. Gang Bao developed the IONP, provided expertise on IONP synthesis and characterization, help with study design, and funding to cover the IONP synthesis and characterization. 
John C. Bischof provided IONP heating expertise, help with study design, the inductive coil for heating, and funding to cover salary and supplies.

\section{Competing Interests}

The authors have declared that no competing interest exists.

\section{Data Availability}

The datasets generated during and/or analyzed during the current study are available from the corresponding author on reasonable request.

\section{References}

$1 \quad$ Kut, C. et al. Preliminary study of injury from heating systemically delivered, nontargeted dextransuperparamagnetic iron oxide nanoparticles in mice. Nanomedicine (London, England) 7, 16971711, doi:10.2217/nnm.12.65 (2012).

2 Etheridge, M. L. \& Bischof, J. C. Optimizing Magnetic Nanoparticle Based Thermal Therapies Within the Physical Limits of Heating. Annals of Biomedical Engineering 41, 78-88, doi:10.1007/s10439-012-0633-1 (2013).

3 Rohrer, M., Bauer, H., Mintorovitch, J., Requardt, M. \& Weinmann, H.-J. Comparison of Magnetic Properties of MRI Contrast Media Solutions at Different Magnetic Field Strengths. Investigative Radiology 40 (2005).

4 Zhang, J. et al. Quantification and biodistribution of iron oxide nanoparticles in the primary clearance organs of mice using T1 contrast for heating. Magnetic Resonance in Medicine 78, 702712, doi:10.1002/mrm.26394 (2017).

5 Manuchehrabadi, N. et al. Improved tissue cryopreservation using inductive heating of magnetic nanoparticles. Science Translational Medicine 9 (2017).

6 Hoopes, P. J. et al. In Vivo Imaging and Quantification of Iron Oxide Nanoparticle Uptake and Biodistribution. Proceedings of SPIE 8317, 83170R, doi:10.1117/12.916097 (2012).

7 Ring, H. L., Bischof, J. C. \& Garwood, M. in Handbook - RF Safety (ed D. Shrivastava) (eMagRes, 2019).

8 Smolensky, E. D., Park, H.-Y. E., Berquó, T. S. \& Pierre, V. C. Surface functionalization of magnetic iron oxide nanoparticles for MRI applications - effect of anchoring group and ligand exchange protocol. Contrast Media \& Molecular Imaging 6, 189-199, doi:10.1002/cmmi.417 (2011).

9 Tong, S., Quinto, C. A., Zhang, L., Mohindra, P. \& Bao, G. Size-Dependent Heating of Magnetic Iron Oxide Nanoparticles. ACS Nano 11, 6808-6816, doi:10.1021/acsnano.7b01762 (2017).

10 Tong, S., Hou, S., Zheng, Z., Zhou, J. \& Bao, G. Coating Optimization of Superparamagnetic Iron Oxide Nanoparticles for High T2 Relaxivity. Nano Letters 10, 4607-4613, doi:10.1021/nl102623x (2010).

11 Cassim, S. M., Giustini, A. J., Baker, I. \& Hoopes, P. J. Development of Novel Magnetic Nanoparticles for Hyperthermia Cancer Therapy. Proceedings of SPIE 7901, 790115, doi:10.1117/12.876514 (2011).

12 Hoopes, P. J. et al. in SPIE Proceedings.

13 Pearce, J., Giustini, A., Stigliano, R. \& Jack Hoopes, P. Magnetic Heating of Nanoparticles: The Importance of Particle Clustering to Achieve Therapeutic Temperatures. Journal of Nanotechnology in Engineering and Medicine 4, 0110071-01100714, doi:10.1115/1.4024904 (2013).

14 Bordelon, D. E. et al. Magnetic nanoparticle heating efficiency reveals magneto-structural differences when characterized with wide ranging and high amplitude alternating magnetic fields. Journal of Applied Physics 109, 124904, doi:doi:http://dx.doi.org/10.1063/1.3597820 (2011). 
15 Dennis, C. L. et al. The influence of collective behavior on the magnetic and heating properties of iron oxide nanoparticles. Journal of Applied Physics 103, 07A319, doi:10.1063/1.2837647 (2008).

16 Wabler, M. et al. Magnetic resonance imaging contrast of iron oxide nanoparticles developed for hyperthermia is dominated by iron content. International journal of hyperthermia : the official journal of European Society for Hyperthermic Oncology, North American Hyperthermia Group 30, 192-200, doi:10.3109/02656736.2014.913321 (2014).

17 Wu, W., Wu, Z., Yu, T., Jiang, C. \& Kim, W.-S. Recent progress on magnetic iron oxide nanoparticles: synthesis, surface functional strategies and biomedical applications. Science and Technology of Advanced Materials 16, 023501 (2015).

18 Smolensky, E. D. et al. Scaling Laws at the Nano Size: The Effect of Particle Size and Shape on the Magnetism and Relaxivity of Iron Oxide Nanoparticle Contrast Agents. Journal of materials chemistry. B, Materials for biology and medicine 1, 2818-2828, doi:10.1039/C3TB00369H (2013).

19 Zhang, J. et al. Quantifying iron-oxide nanoparticles at high concentration based on longitudinal relaxation using a three-dimensional SWIFT look-locker sequence. Magnetic Resonance in Medicine 71, 1982-1988, doi:10.1002/mrm.25181 (2014).

20 Clement, O., Siauve, N., Cuénod, C.-A. \& Frija, G. Liver Imaging With Ferumoxides (Feridex®): Fundamentals, Controversies, and Practical Aspects. 9, 167-182 (1998).

21 Wang, Y.-X. J. Current status of superparamagnetic iron oxide contrast agents for liver magnetic resonance imaging. World Journal of Gastroenterology 21, 13400-13402, doi:10.3748/wjg.v21.i47.13400 (2015).

22 Wáng, Y. X. J. \& Idée, J.-M. A comprehensive literatures update of clinical researches of superparamagnetic resonance iron oxide nanoparticles for magnetic resonance imaging. Quantitative Imaging in Medicine and Surgery 7, 88-122, doi:10.21037/qims.2017.02.09 (2017).

23 Kuhlpeter, R. et al. R2 and R2* Mapping for Sensing Cell-bound Superparamagnetic Nanoparticles: In Vitro and Murine in Vivo Testing. Radiology 245, 449-457, doi:10.1148/radiol.2451061345 (2007).

24 Wei, H. et al. Exceedingly small iron oxide nanoparticles as positive MRI contrast agents. Proceedings of the National Academy of Sciences of the United States of America 114, 2325-2330, doi:10.1073/pnas.1620145114 (2017).

25 Etheridge, M. L. et al. Accounting for biological aggregation in heating and imaging of magnetic nanoparticles. Technology 02, 214-228, doi:10.1142/S2339547814500198 (2014).

26 Ring, H. L. et al. Establishing the overlap of IONP quantification with echo and echoless MR relaxation mapping. Magnetic Resonance in Medicine 79, 1420-1428, doi:10.1002/mrm.26800 (2017).

27 Zhou, R. et al. SWIFT detection of SPIO-labeled stem cells grafted in the myocardium. Magnetic Resonance in Medicine 63, 1154-1161, doi:10.1002/mrm.22378 (2010).

28 Wang, L., Corum, C. A., Idiyatullin, D., Garwood, M. \& Zhao, Q. T(1) Estimation for Aqueous Iron Oxide Nanoparticle Suspensions Using a Variable Flip Angle SWIFT Sequence. Magnetic resonance in medicine : official journal of the Society of Magnetic Resonance in Medicine / Society of Magnetic Resonance in Medicine 70, 10.1002/mrm.24831, doi:10.1002/mrm.24831 (2013).

29 Wang, L. et al. Ultrashort Echo Time (UTE) imaging of receptor targeted magnetic iron oxide nanoparticles in mouse tumor models. Journal of Magnetic Resonance Imaging 40, 1071-1081, doi:10.1002/jmri.24453 (2014).

30 Gharagouzloo, C. A., McMahon, P. N. \& Sridhar, S. Quantitative contrast-enhanced MRI with superparamagnetic nanoparticles using ultrashort time-to-echo pulse sequences. Magnetic Resonance in Medicine 74, 431-441, doi:10.1002/mrm.25426 (2015).

31 Mornet, S., Vasseur, S., Grasset, F. \& Duguet, E. Magnetic nanoparticle design for medical diagnosis and therapy. Journal of Materials Chemistry 14, 2161-2175, doi:10.1039/B402025A (2004). 
32 Rosensweig, R. E. Heating magnetic fluid with alternating magnetic field. Journal of Magnetism and Magnetic Materials 252, 370-374, doi:http://dx.doi.org/10.1016/S0304-8853(02)00706-0 (2002).

33 Dennis, C. L. et al. Nearly complete regression of tumors via collective behavior of magnetic nanoparticles in hyperthermia. Nanotechnology 20, 395103 (2009).

34 Giustini, A. J., Ivkov, R. \& Hoopes, P. J. Magnetic nanoparticle biodistribution following intratumoral administration. Nanotechnology 22, 345101-345101, doi:10.1088/09574484/22/34/345101 (2011).

35 Murase, K., Takata, H., Takeuchi, Y. \& Saito, S. Control of the temperature rise in magnetic hyperthermia with use of an external static magnetic field. Physica Medica 29, 624-630, doi:https://doi.org/10.1016/j.ejmp.2012.08.005 (2013).

36 Tay, Z. W. et al. Magnetic Particle Imaging-Guided Heating in Vivo Using Gradient Fields for Arbitrary Localization of Magnetic Hyperthermia Therapy. ACS Nano 12, 3699-3713, doi:10.1021/acsnano.8b00893 (2018).

37 Gneveckow, U. et al. Description and characterization of the novel hyperthermia-and thermoablation-system MFH300F for clinical magnetic fluid hyperthermia. Medical physics 31, 1444-1444 (2004).

38 Hurley, K. R. et al. Predictable Heating and Positive MRI Contrast from a Mesoporous SilicaCoated Iron Oxide Nanoparticle. Mol. Pharm. 13, 2172-2183 (2016).

39 Carrey, J., Mehdaoui, B. \& Respaud, M. Simple models for dynamic hysteresis loop calculations of magnetic single-domain nanoparticles: Application to magnetic hyperthermia optimization. Journal of Applied Physics 109, 083921, doi:10.1063/1.3551582 (2011).

40 Jeun, M. et al. Physical Parameters to Enhance AC Magnetically Induced Heating Power of Ferrite Nanoparticles for Hyperthermia in Nanomedicine. IEEE Transactions on Nanotechnology 12, 314322, doi:10.1109/TNANO.2013.2247414 (2013).

41 Bonvin, D. et al. Tuning Properties of Iron Oxide Nanoparticles in Aqueous Synthesis without Ligands to Improve MRI Relaxivity and SAR. Nanomaterials 7, doi:10.3390/nano7080225 (2017).

42 Mohapatra, J., Mitra, A., Aslam, M. \& Bahadur, D. Octahedral-Shaped Fe3O4 Nanoparticles With Enhanced Specific Absorption Rate and Relaxivity. IEEE Transactions on Magnetics 51, 1-3, doi:10.1109/TMAG.2015.2439213 (2015).

43 Lartigue, L. et al. Water-Dispersible Sugar-Coated Iron Oxide Nanoparticles. An Evaluation of their Relaxometric and Magnetic Hyperthermia Properties. Journal of the American Chemical Society 133, 10459-10472, doi:10.1021/ja111448t (2011).

44 Bulte, J. W. M. \& Daldrup-Link, H. E. Clinical Tracking of Cell Transfer and Cell Transplantation: Trials and Tribulations. Radiology 289, 604-615, doi:10.1148/radiol.2018180449 (2018).

45 Liang, C., Wang, C. \& Liu, Z. Stem Cell Labeling and Tracking with Nanoparticles. Particle \& Particle Systems Characterization 30, 1006-1017, doi:10.1002/ppsc.201300199 (2013).

46 Idiyatullin, D., Corum, C., Park, J.-Y. \& Garwood, M. Fast and Quiet MRI using a Sweep Radiofrequency. Journal of Magnetic Resonance 181, 342-349, doi:10.1016/j.jmr.2006.05.014 (2006).

47 Girard, O. M. et al. Optimization of Iron Oxide Nanoparticle Detection Using Ultrashort TE Pulse Sequences: Comparison of T(1), T(2)* and Synergistic T(1)-T(2)* Contrast Mechanisms. Magnetic resonance in medicine : official journal of the Society of Magnetic Resonance in Medicine / Society of Magnetic Resonance in Medicine 65, 1649-1660, doi:10.1002/mrm.22755 (2011).

48 Idiyatullin, D., Corum, C. A. \& Garwood, M. Multi-Band-SWIFT. Journal of Magnetic Resonance 251, 19-25, doi:http://dx.doi.org/10.1016/j.jmr.2014.11.014 (2015).

49 Gillis, P. \& Koenig, S. H. Transverse relaxation of solvent protons induced by magnetized spheres: Application to ferritin, erythrocytes, and magnetite. Magnetic Resonance in Medicine 5, 323-345, doi:10.1002/mrm.1910050404 (1987).

50 Gillis, P., Roch, A. \& Brooks, R. A. Corrected Equations for Susceptibility-InducedT2-Shortening. Journal of Magnetic Resonance 137, 402-407, doi:https://doi.org/10.1006/jmre.1998.1691 (1999). 
51 Laurent, S. et al. Magnetic Iron Oxide Nanoparticles: Synthesis, Stabilization, Vectorization, Physicochemical Characterizations, and Biological Applications. Chemical Reviews 108, 20642110, doi:10.1021/cr068445e (2008).

52 Dennis Cindi, L. et al. Internal Magnetic Structure of Nanoparticles Dominates Time-Dependent Relaxation Processes in a Magnetic Field. Advanced Functional Materials 25, 4300-4311, doi:10.1002/adfm.201500405 (2015).

53 Hurley, K. R., Lin, Y.-s., Zhang, J., Egger, S. M. \& Haynes, C. L. Effects of Mesoporous Silica Coating and Post-Synthetic Treatment on the Transverse Relaxivity of Iron Oxide Nanoparticles. Chemistry of materials : a publication of the American Chemical Society 25, 1968-1978, doi:10.1021/cm400711h (2013).

54 Fish, W. W. Rapid colorimetric micromethod for the quantitation of complexed iron in biological samples. Methods in enzymology 158, 357-364, doi:10.1016/0076-6879(88)58067-9 (1988).

55 Lahiri, B. B., Ranoo, S. \& Philip, J. Uncertainties in the estimation of specific absorption rate during radiofrequency alternating magnetic field induced non-adiabatic heating of ferrofluids. Journal of Physics D: Applied Physics 50, 455005 (2017). 


\section{Tables}

Table 1: IONP

Diameter

\begin{tabular}{c|c}
\hline $\begin{array}{c}\text { IONP } \\
\text { size }\end{array}$ & $\begin{array}{c}\text { Core diameter } \\
(\mathrm{nm}, \text { mean } \pm \\
\text { st. dev. })\end{array}$ \\
\hline 5 & $4.9 \pm 0.5$ \\
6 & $5.6 \pm 0.9$ \\
11 & $10.6 \pm 1.3$ \\
16 & $16.1 \pm 1.2$ \\
19 & $18.9 \pm 1.7$ \\
33 & $33.0 \pm 3.8$
\end{tabular}

\section{Figures}

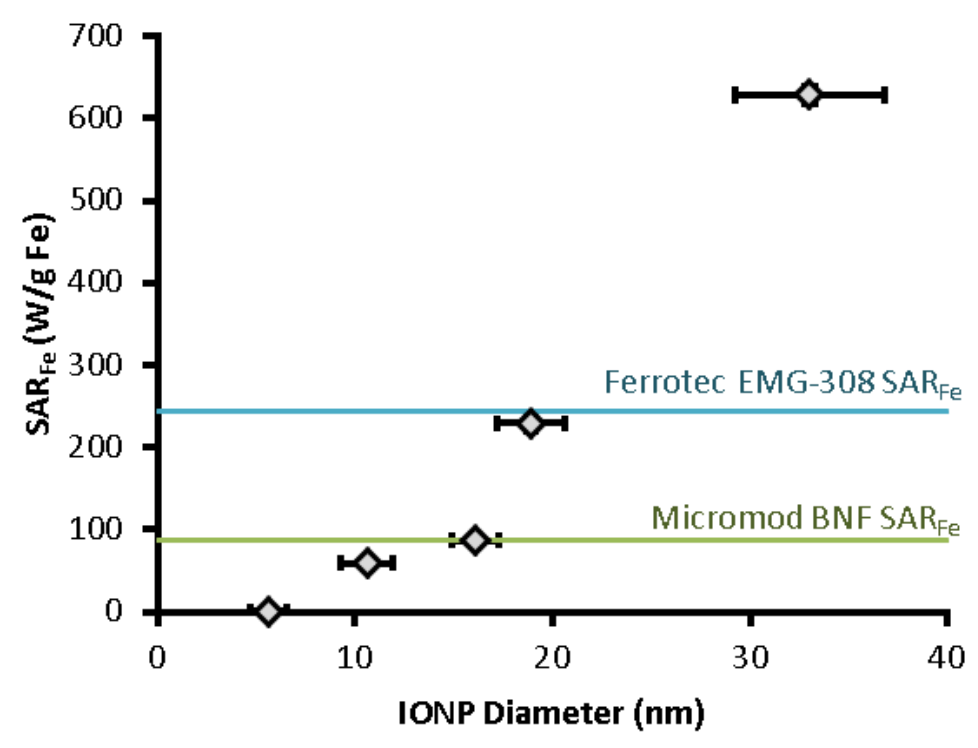

Figure 1: Heating characteristics as a function of IONP size. SARFe is observed to increase with IONP diameter. Lines are drawn for comparison of Ferrotec EMG-308 and Micromod BNF SAR Fe as previously measured by Etheridge et. al. ${ }^{2}$ and do not represent IONP diameter. The $33 \mathrm{~nm}$ IONP produced approximately three-fold greater heat than that of Ferrotec $(p<0.001)$. Error bars indicate the standard deviation $(n=3)$. Error bars which are not visible are smaller than the data point. 

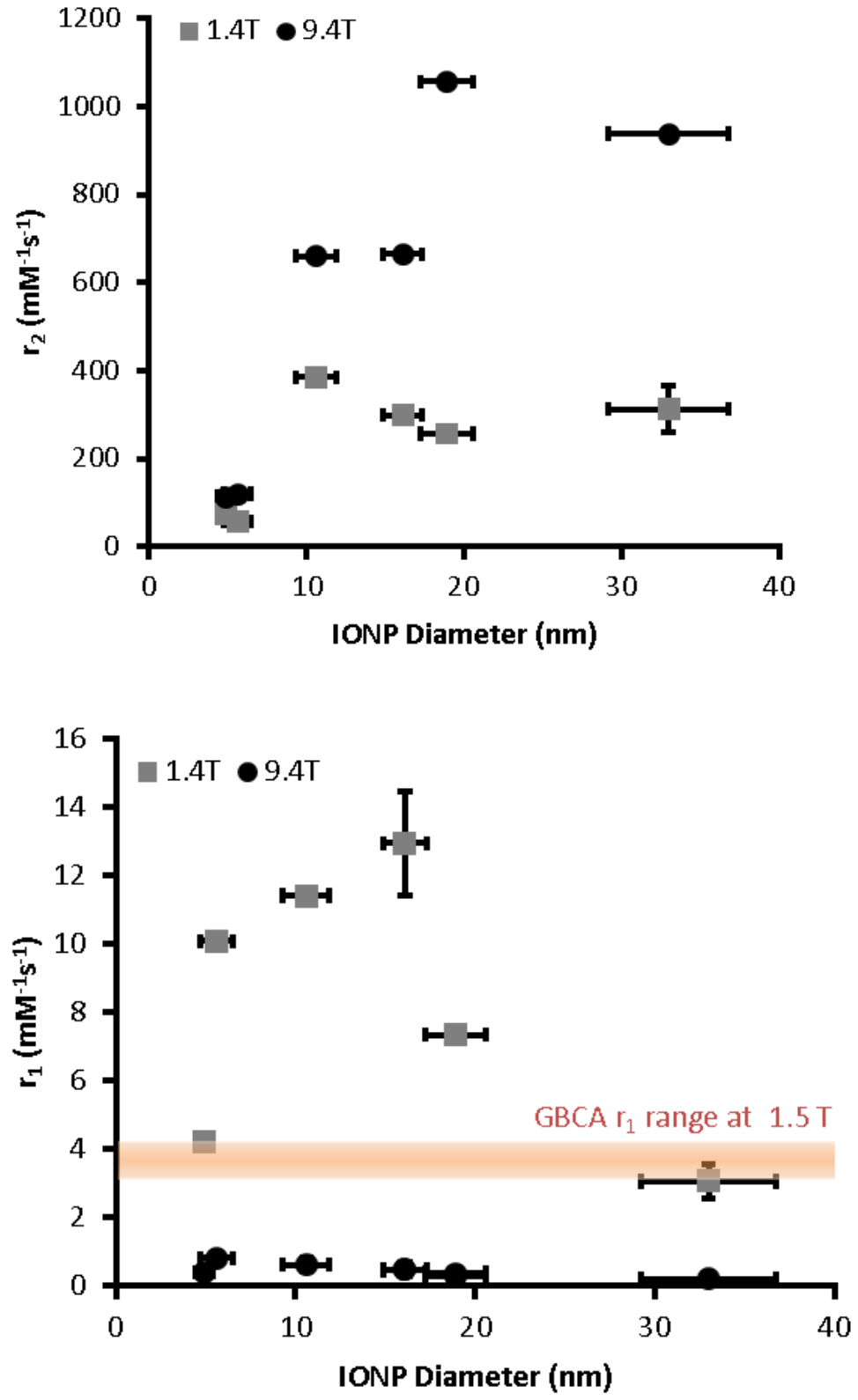

Figure 2: Relaxivity $\left(r_{2}\right)$ is shown as a function of IONP diameter. At both $1.4 \mathrm{~T}$ (grey squares) and $9.4 T$ (black circles), $r_{2}$ increases with IONP diameter. Vertical error bars indicate standard error of the mean $(n=3)$, which in most cases is smaller than the data point. Horizontal data points indicate the standard deviation of the IONP diameter.
Figure 3: rI relaxivity is shown as a function of IONP diameter. At both $1.4 \mathrm{~T}$ (grey squares) and $9.4 T$ (black circles), $r_{I}$ has a local maximum and then decreases with IONP diameter. Vertical error bars indicate standard error of measurement $(n=3)$, which in many cases is smaller than the data point. Horizontal error bars indicate the standard deviation of the IONP diameter. The line shown indicates a comparison with clinically used GBCAs and do not represent diameter ${ }^{3}$. 

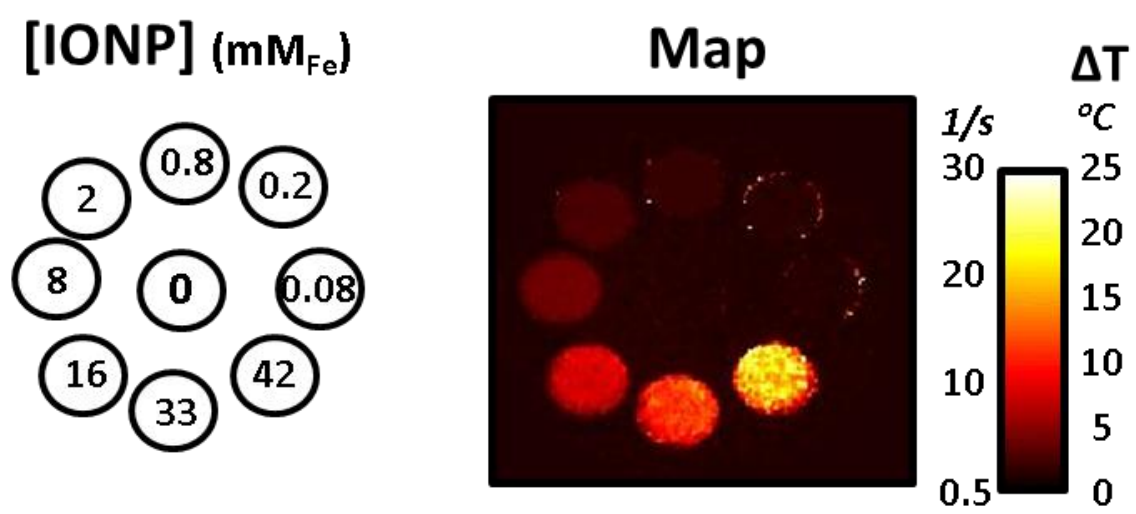

Figure 4: A demonstration of converting an $R_{l}$ map to expected temperature changes (UT) after 3 minutes of heating. This specific dataset was for the $16 \mathrm{~nm}$ IONPs at concentrations ranging from 0.08 to $42 \mathrm{mM}_{\mathrm{Fe}}\left(0.005\right.$ to $2.5 \mathrm{mg}$ Fe/mL). The $R_{1}$ map was measured at $9.4 T$ with the $\Delta T$ map calculated using the calibration curve created by heating these samples. The temperature change for IONP concentrations $\geq 16 \mathrm{mMFe}_{\mathrm{Fe}}(1 \mathrm{mg} \mathrm{Fe} / \mathrm{mL})$ produce a sufficient temperature change to theoretically cause tissue damage ${ }^{1,2}$. 